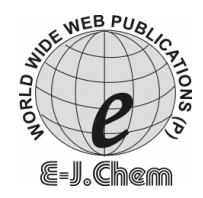

ISSN: 0973-4945; CODEN ECJHAO

E-Journal of Chemistry

http://www.e-journals.net

2009, 6(4), 1193-1199

\title{
Evaluation of the Phytochemical Content of Some Edible Grains Marketed in Nigeria
}

\author{
S. A. ODOEMELAM and C.I. OSU \\ Department of Chemistry, \\ Michael Okpara University of Agriculture, Umudike, \\ P.M.B 7267, Umuahia, Abia State, Nigeria. \\ saodoemelam@yahoo.com
}

Received 18 August 2008; Accepted 15 January 2009

\begin{abstract}
Astract: Five food grains (groundnut, millet, wheat, guinea corn and breadfruit) commonly consumed in Nigeria were evaluated for their phytochemical contents. The results revealed that the grains contained $0.60-1.24 \%$ alkaloid, $0.82-2.27 \%$ flavonoid, $0.03-0.05 \%$ saponin, $0.50-0.60 \%$ tannins, $2.40-2.80 \%$ phenols and $0.17-0.30 \%$ phytic acid. Dietary implications of these constituents are highlighted
\end{abstract}

Keywords: Phytochemicals, Anti-nutritional factors, Food, Grains.

\section{Introduction}

Food grains represent the main supplementary energy source in starchy food-based diets consumed by large segments of people living in Nigeria and many other developing countries. Grains such as millet, wheat, guinea corn etc constitute staple diets of most of the people living in Northern Nigeria while in other parts of the country they are eaten as complement foods. Generally grains have found good applications in food products and animal feeds. They are good sources of metabolizable energy, dietary minerals, proteins, carbohydrate and vitamins.

Food grain legumes e.g. beans, lentils, groundnuts, breadfruit, etc. have high nutrient bioavailability, particularly high protein, which allows them to serve as excellent protein supplements to cereal grains ${ }^{1}$. According to Philips and McWaters ${ }^{2}$ cereals and food legumes provide a great part of dietary nutrients for large populations of people living in developing countries where protein-rich foods are expensive.

In addition to their nutritionally useful constituents, grains have been found to contain phytochemicals and anti-nutritional factors, which constitute a great health hazard. Thus the nutritional value of food grains can be divided into positive factors, which are nutritionally 
beneficial and the negative factors, which are non-beneficial. Ahmed et $a l^{3}$., have identified the positive factors as high protein and fibre while the negative factors include anti-nutritional constituents like polyphenols, tannin, phytate, enzyme (Trypsin) inhibitors etc.

According to Aletor and Adeogun ${ }^{4}$, the presence of inherent anti-nutritional components in plants is a major factor affecting the nutritional value of foods and feeds. The objective of this study was therefore to estimate the phytochemical constituents and anti-nutritional factors present in selected food grains from markets in the Niger Delta region of Nigeria.

\section{Experimental}

The millet, wheat, Guinea corn, groundnut and breadfruit used in this study were randomly purchased directly from major markets in Abia, Akwa Ibom, Bayelsa, Cross River, Delta, Edo, Imo, Rivers and Ondo States of Nigeria. The grain samples were sorted to remove foreign materials, washed, dried and ground into uniform powder using Author Thomas machine and stored in labeled air-tight bottles till required for analysis.

\section{Phytochemical analyses}

Alkaloid contents of the samples were estimated by the alkaline precipitation method described by Harborne ${ }^{5}$. Total phytate contents of the samples were determined using the spectrophotometric method described by Hang and Lantzsch ${ }^{6}$ while saponins were quantified according to the method described Obadoni and Ochuko ${ }^{7}$. Flavonoids were determined following the method of Bohm and Kocipai-Abyazan ${ }^{8}$. Total phenols were determined according to the Prucian Blue spectrophotometic method described by Price and Butler $^{9}$ while tannins were estimated using the Folin-Dennis spectrophotometric method described by Pearson ${ }^{10}$.

\section{Statistical analysis}

Three replicate samples of each food grain from each of the states in the study area were analyzed and values were averaged. Data were assessed by analysis of variance (ANOVA) and Duncan's multiple range tests with a probability $\mathrm{p} \leq 0.05$.

\section{Results and Discussion}

The phytochemicals determined in the food items investigated are presented in Tables 1-6. The range of concentration of alkaloids obtained were groundnut, 1.12-1.36\%, millet, 0.50 $0.78 \%$, wheat, $0.52-0.68 \%$, guinea corn, $0.65-0.83 \%$ and breadfruit, $0.66-0.92 \%$ (Table 1). The highest alkaloid content of $1.36 \%$ was obtained from groundnut from Abia State while the lowest concentration of $0.50 \%$ was recorded for millet from Cross River State. Groundnut had the highest concentration of alkaloids with a mean value of $1.24 \pm 0.08 \%$ while wheat had the lowest concentration with a mean value of $0.61 \pm 0.06 \%$. Groundnut samples from all the states showed significant differences in their alkaloid contents at $\mathrm{p}<0.05$ while the other food commodities showed some similarities in the alkaloid contents of samples from 2 or 3 states. For instance there was no significant difference between the concentrations of alkaloids in breadfruit samples from Akwa Ibom, Cross River and Rivers States. Alkaloids affect a lot of metabolic activities in the body and most of them possess dramatic physiological activities hence they are widely used in medicine ${ }^{5}$. Alkaloids are toxic to man. Certain plant alkaloids cause serious intoxications in animals and humans and are often mutagenic ${ }^{3,4}$. 
Table 1. Alkaloid content of grains, (\%).

\begin{tabular}{cccccc}
\hline State & Groundnut & Millet & Wheat & Guinea corn & Bread fruit \\
\hline Abia & $1.36^{\mathrm{a}} \pm 0.0 .04$ & $0.78^{\mathrm{hi}} \pm 0.04$ & $0.68^{\mathrm{mo}} \pm 0.07$ & $0.77^{\mathrm{ik}} \pm 0.07$ & $0.92^{\mathrm{g}} \pm 0.05$ \\
Imo & $1.31^{\mathrm{ab}} \pm 0.03$ & $0.73^{\mathrm{jm}} \pm 0.06$ & $0.67^{\mathrm{mq}} \pm 0.01$ & $0.75^{\mathrm{i}} \pm 0.06$ & $0.81^{\mathrm{hi}} \pm 0.03$ \\
Akwa Ibom & $1.15^{\mathrm{ef}} \pm 0.04$ & $0.53^{\mathrm{tv}} \pm 0.04$ & $0.61^{\mathrm{ps}} \pm 0.03$ & $0.73^{\mathrm{jm}} \pm 0.03$ & $0.73^{\mathrm{il}} \pm 0.03$ \\
Cross River & $1.12^{\mathrm{f}} \pm 0.04$ & $0.50^{\mathrm{v}} \pm 0.05$ & $0.63^{\mathrm{or}} \pm 0.02$ & $0.83^{\mathrm{h}} \pm 0.03$ & $0.76^{\mathrm{il}} \pm 0.03$ \\
Rivers & $1.30^{\mathrm{b}} \pm 0.02$ & $0.54^{\mathrm{tv}} \pm 0.06$ & $0.52^{\mathrm{uv}} \pm 0.01$ & $0.80^{\mathrm{hi}} \pm 0.10$ & $0.76^{\mathrm{il}} \pm 0.04$ \\
Delta & $1.26^{\mathrm{bc}} \pm 0.03$ & $0.63^{\mathrm{or}} \pm 0.01$ & $0.54^{\mathrm{tu}} \pm 0.01$ & $0.70^{\mathrm{ln}} \pm 0.01$ & $0.72^{\mathrm{jm}} \pm 0.01$ \\
Edo & $1.23^{\mathrm{cd}} \pm 0.03$ & $0.63^{\mathrm{or}} \pm 0.02$ & $0.61^{\mathrm{ps}} \pm 0.02$ & $0.81^{\mathrm{hi}} \pm 0.05$ & $0.71^{\mathrm{kn}} \pm 0.02$ \\
Ondo & $1.19^{\mathrm{de}} \pm 0.02$ & $0.65^{\mathrm{nq}} \pm 0.03$ & $0.56^{\mathrm{su}} \pm 0.01$ & $0.65^{\mathrm{nq}} \pm 0.05$ & $066^{\mathrm{nq}} \pm 0.01$ \\
Mean & $1.24^{\mathrm{a}} \pm 0.08$ & $0.63^{\mathrm{c}} \pm 0.10$ & $0.06^{\mathrm{d}} \pm 0.06$ & $0.75^{\mathrm{b}} \pm 0.07$ & $0.76^{\mathrm{b}} \pm 0.08$ \\
\hline
\end{tabular}

Values are mean \pm S.D of three determinations. Values not sharing common superscript letter $(s)$ in a column are significantly different at $P<0.05$.

The concentrations of flavonoids from the samples examined are presented in Table 2. Mean concentrations of $0.82 \pm 0.13 \%, 1.56 \pm 0.15 \%, 1.78 \pm 0.12 \%, 1.48- \pm 0.09 \%$ and $2.27 \pm 0.55 \%$ were recorded for groundnut, millet, wheat, guinea corn and breadfruit, respectively.

Table 2. Flavonoid content of grains, (\%).

\begin{tabular}{cccccc}
\hline State & Groundnut & Millet & Wheat & Guinea corn & Bread fruit \\
\hline Abia & $0.65^{\mathrm{q}} \pm 0.02$ & $1.60^{\mathrm{gi}} \pm 0.09$ & $1.86^{\mathrm{f}} \pm 0.05$ & $1.41^{\mathrm{kn}} \pm 0.01$ & $3.16^{\mathrm{a}} \pm 0.09$ \\
Imo & $0.699^{\mathrm{bq}} \pm 0.02$ & $1.58^{\mathrm{hi}} \pm 0.13$ & $1.85^{\mathrm{f}} \pm 0.15$ & $1.49^{\mathrm{jm}} \pm 0.04$ & $2.04^{\mathrm{e}} \pm 0.04$ \\
Akwa Ibom & $0.95^{\mathrm{o}} \pm 0.02$ & $1.77^{\mathrm{f}} \pm 0.05$ & $1.82^{\mathrm{f}} \pm 0.13$ & $1.47^{\mathrm{in}} \pm 0.06$ & $2.83^{\mathrm{b}} \pm 0.03$ \\
Cross River & $0.73^{\mathrm{pq}} \pm 0.02$ & $1.73^{\mathrm{fl}} \pm 0.04$ & $1.77^{\mathrm{f}} \pm 0.01$ & $1.36^{\mathrm{mn}} \pm 0.04$ & $2.62^{\mathrm{c}} \pm 0.13$ \\
Rivers & $0.67 \pm 0.01$ & $1.37 \pm 0.04$ & $1.76^{\mathrm{f}} \pm 0.07$ & $1.39^{\mathrm{ln}} \pm 0.09$ & $2.26^{\mathrm{d}} \pm 0.07$ \\
Bayelsa & $0.82^{\mathrm{op}} \pm 0.02$ & $1.33^{\mathrm{n}} \pm 0.04$ & $1.76^{\mathrm{fh}} \pm 0.01$ & $1.59^{\mathrm{hj}} \pm 0.03$ & $2.26^{\mathrm{d}} \pm 0.18$ \\
Delta & $0.97^{\mathrm{o}} \pm 0.02$ & $1.56^{\mathrm{ik}} \pm 0.03$ & $1.76^{\mathrm{fh}} \pm 0.01$ & $1.59^{\mathrm{ik}} \pm 0.03$ & $2.29^{\mathrm{d}} \pm 0.18$ \\
Edo & $0.91^{\mathrm{o}} \pm 0.02$ & $1.57^{\mathrm{ik}} \pm 0.02$ & $1.71^{\mathrm{fl}} \pm 0.02$ & $1.48^{\mathrm{ik}} \pm 0.08$ & $1.60^{\mathrm{d}} \pm 0.12$ \\
Ondo & $0.97^{\circ} \pm 0.02$ & $1.54^{\mathrm{hj}} \pm 0.02$ & $1.75^{\mathrm{fh}} \pm 0.28$ & $1.57^{\mathrm{in}} \pm 0.04$ & $1.36^{\mathrm{gj}} \pm 0.0$ \\
Mean & $0.82^{\mathrm{e}} \pm 0.13$ & $1.56^{\mathrm{c}} \pm 0.15$ & $1.78^{\mathrm{b}} \pm 0.12$ & $1.48^{\mathrm{d}} \pm 0.09$ & $2.27^{\mathrm{a}} \pm 0.55$ \\
\hline
\end{tabular}

Values are mean \pm S.D of three determinations. Values not sharing common superscript letter $(s)$ in a column are significantly different at $P<0.05$. 
The highest flavonoid content of $3.16 \%$ was obtained from breadfruit from Abia State while the lowest concentration of $0.65 \%$ was recorded for groundnut from Abia State. Breadfruit had the highest flavonoid content (overall mean $=2.27 \pm 0.55$ ) compared to the other food items studied. Plant Flavonoids have attracted attention as potentially important dietary cancer chemoprotective agents ${ }^{11}$. In addition, the possible anti tumor action of certain flavonoids has also generated interest ${ }^{12-14}$. Perhaps the grains may provide the necessary dietary bioflavonoids required to prevent cancer and the growth of certain tumor in humans when adequate quantities are consumed regularly. Flavonoids have been reported to possess certain biological properties such as antibacterial, antitoxic and anti-inflammatory activities and often function as strong antioxidants, free radical scavengers and metal chelators ${ }^{15,16}$.

The saponins contents of the samples are presented in Table 3 . The phytochemical score of the samples showed that saponins ranked last. The mean concentrations of saponins obtained were $0.04 \pm 0.02 \%, 0.03 \pm 0.02 \%, 0.05 \pm 0.03 \%, 0.03 \pm 0.02 \%$ and $0.05 \pm 0.03 \%$ for groundnut, millet, wheat, guinea corn and breadfruit, respectively. The highest saponins content of $0.11 \%$ was recorded for wheat from Ondo State and the lowest concentration of $0.01 \%$ was obtained from groundnut (Ondo State) and guinea corn (Abia and Edo States). From the results obtained, breadfruit and wheat had the highest overall mean saponin compared to the other food items studied.Plant saponins are components of glycosides and are often referred to as "natural detergents" because of their foamy nature. They are known to have both beneficial and deleterious properties depending on their concentration. Saponins have been reported to possess anticarcinogenic properties, immune modulation activities and regulation of cell proliferation as well as health benefits such as inhibition of the growth of cancer cells and cholesterol lowering activity ${ }^{16}$.

Table 3. Saponins content of grains, \%.

\begin{tabular}{cccccc}
\hline State & Groundnut & Millet & Wheat & Guinea corn & Bread fruit \\
\hline Abia & $0.03^{\mathrm{gi}} \pm 0.01$ & $0.04^{\mathrm{fl}} \pm 0.01$ & $0.02^{\mathrm{il}} \pm 0.01$ & $0.01^{\mathrm{l}} \pm 0.01$ & $0.05^{\mathrm{di}} \pm 0.01$ \\
Imo & $0.02^{\mathrm{ji}} \pm 0.01$ & $0.02^{\mathrm{hi}} \pm 0.01$ & $0.06^{\mathrm{cf}} \pm 0.01$ & $0.05^{\mathrm{di}} \pm 0.01$ & $0.02^{\mathrm{il}} \pm 0.01$ \\
Akwa Ibom & $0.04^{\mathrm{fl}} \pm 0.01$ & $0.04^{\mathrm{fi}} \pm 0.04$ & $0.03^{\mathrm{gi}} \pm 0.02$ & $0.04^{\mathrm{fi}} \pm 0.03$ & $0.05^{\mathrm{di}} \pm 0.0$ \\
Cross River & $0.04^{\mathrm{fl}} \pm 0.02$ & $0.02^{\mathrm{ji}} \pm 0.01$ & $0.04^{\mathrm{fi}} \pm 0.03$ & $0.03^{\mathrm{gi}} \pm 0.02$ & $0.02^{\mathrm{ji}} \pm 0.01$ \\
Rivers & $0.06^{\mathrm{cf}} \pm 0.01$ & $0.04^{\mathrm{fl}} \pm 0.02$ & $0.03^{\mathrm{gl}} \pm 0.01$ & $0.04^{\mathrm{fl}} \pm 0.01$ & $0.06^{\mathrm{cf}} \pm 0.03$ \\
Bayelsa & $0.05^{\mathrm{di}} \pm 0.01$ & $0.02^{\mathrm{jl}} \pm 0.01$ & $0.09^{\mathrm{ab}} \pm 0.01$ & $0.04^{\mathrm{fl}} \pm 0.02$ & $0.08^{\mathrm{bc}} \pm 0.02$ \\
Delta & $0.05^{\mathrm{di}} \pm 0.02$ & $0.03^{\mathrm{gl}} \pm 0.03$ & $0.07^{\mathrm{bd}} \pm 0.01$ & $0.03^{\mathrm{gl}} \pm 0.02$ & $0.06^{\mathrm{cf}} \pm 0.02$ \\
Edo & $0.04^{\mathrm{fl}} \pm 0.01$ & $0.03^{\mathrm{gl}} \pm 0.01$ & $0.02^{\mathrm{jl}} \pm 0.02$ & $0.01^{1} \pm 0.01$ & $0.08^{\mathrm{bc}} \pm 0.02$ \\
Ondo & $0.01^{\mathrm{b}} \pm 0.00$ & $0.04^{\mathrm{fl}} \pm 0.04$ & $0.11^{\mathrm{a}} \pm 0.01$ & $0.02^{\mathrm{jl}} \pm 0.01$ & $0.03^{\mathrm{gl}} \pm 0.01$ \\
Mean & $0.04^{\mathrm{b}} \pm 0.02$ & $0.03^{\mathrm{b}} \pm 0.02$ & $0.05^{\mathrm{a}} \pm 0.03$ & $0.03^{\mathrm{b}} \pm 0.02$ & $0.05^{\mathrm{a}} \pm 0.03$ \\
\hline
\end{tabular}

Values are mean \pm S.D of three determinations. Values not sharing common superscript letter $(s)$ in a column are significantly different at $P<0.05$. 
Results of the concentrations of tannins are presented in Table 4. The range of concentrations of tannins obtained were groundnut $0.57-0.64 \%$, millet $0.48-053 \%$, wheat $0.46-0.55$, guinea corn $0.49-0.58$ and bread fruit $0.49-0.61 \%$. The highest tannins concentration of $0.64 \%$ was recorded for groundnut from Imo State. The lowest tannins level of $0.43 \%$ was obtained from wheat from Bayelsa State. Groundnut had the highest mean concentration $(0.60 \pm 0.03 \%)$ of tannins compared to the other food items studied. Osagie and $\mathrm{Eka}^{17}$ reported that tannins may decrease protein quality by decreasing digestibility and palatability and may cause damage to intestinal tract.

Table 4. Tannins content of grains, (\%).

\begin{tabular}{cccccc}
\hline State & Groundnut & Millet & Wheat & Guinea corn & Bread fruit \\
\hline Abia & $0.60^{\mathrm{a}-\mathrm{e}} \pm 0.02$ & $0.48^{\mathrm{k}-\mathrm{m}} \pm 0.04$ & $0.54_{\mathrm{e}-\mathrm{i}} \pm 0.07$ & $0.52_{\mathrm{g}-\mathrm{l}} \pm 0.05$ & $0.56^{\mathrm{c}-\mathrm{i}} \pm 0.02$ \\
Imo & $0.64^{\mathrm{a}} \pm 0.01$ & $0.50^{\mathrm{i}-\mathrm{l}} \pm 0.01$ & $0.50^{\mathrm{i}-\mathrm{l}} \pm 0.01$ & $0.50_{\mathrm{i}-1} \pm 0.01$ & $0.49^{\mathrm{j}-\mathrm{l}} \pm 0.05$ \\
Akwa Ibom & $0.59^{\mathrm{a}-\mathrm{f}} \pm 0.02$ & $0.49^{\mathrm{j}-\mathrm{l}} \pm 0.03$ & $0.55^{\mathrm{g}-\mathrm{j}} \pm 0.03$ & $0.53^{\mathrm{f}-\mathrm{k}} \pm 0.03$ & $0.60^{\mathrm{a}-\mathrm{e}} \pm 0.04$ \\
Cross River & $0.58^{\mathrm{a}-\mathrm{g}} \pm 0.00$ & $0.53^{\mathrm{g}-\mathrm{k}} \pm 0.02$ & $0.48^{\mathrm{k}-\mathrm{m}} \pm 0.03$ & $0.49^{\mathrm{j}-1} \pm 0.03$ & $0.50^{\mathrm{i}-\mathrm{l}} \pm 0.03$ \\
Rivers & $0.63^{\mathrm{ab}} \pm 0.01$ & $0.52^{\mathrm{g}-\mathrm{l}} \pm 0.02$ & $0.54^{\mathrm{e}-\mathrm{k}} \pm 0.04$ & $0.55^{\mathrm{c}-\mathrm{j}} \pm 0.01$ & $0.50^{\mathrm{j}-\mathrm{i}} \pm 0.02$ \\
Bayelsa & $0.63^{\mathrm{ab}} \pm 0.01$ & $0.53^{\mathrm{g}-\mathrm{k}} \pm 0.01$ & $0.43^{\mathrm{m}} \pm 0.02$ & $0.58^{\mathrm{a}-\mathrm{g}} \pm 0.02$ & $0.55^{\mathrm{e}-\mathrm{j}} \pm 0.02$ \\
Delta & $0.59^{\mathrm{a}-\mathrm{f}} \pm 0.02$ & $0.51^{\mathrm{h}-\mathrm{l}} \pm 0.01$ & $0.50^{\mathrm{i}-\mathrm{j}} \pm 0.01$ & $0.56^{\mathrm{c}-\mathrm{j}} \pm 0.05$ & $0.58^{\mathrm{c}-\mathrm{j}} \pm 0.02$ \\
Edo & $0.60^{\mathrm{a}-\mathrm{d}} \pm 0.01$ & $0.50^{\mathrm{i}-\mathrm{l}} \pm 0.04$ & $0.46^{\mathrm{lm}} \pm 0.03$ & $0.53^{\mathrm{f}-\mathrm{k}} \pm 0.04$ & $0.58^{\mathrm{a}-\mathrm{g}} \pm 0.02$ \\
Ondo & $0.57^{\mathrm{b}-\mathrm{h}} \pm 0.03$ & $0.05^{\mathrm{i}-\mathrm{l}} \pm 0.02$ & $0.52^{\mathrm{g}-\mathrm{l}} \pm 0.02$ & $0.50^{\mathrm{i}-1} \pm 0.04$ & $0.61^{\mathrm{a}-\mathrm{b} \pm 0.02}$ \\
Mean & $0.60^{\mathrm{a}} \pm 0.03$ & $0.51^{\mathrm{d}} \pm 0.03$ & $0.50^{\mathrm{d}} \pm 0.06$ & $0.53^{\mathrm{c}} \pm 0.04$ & $0.55^{\mathrm{b}} \pm 0.05$ \\
\hline
\end{tabular}

Values are mean \pm S.D of three determinations. Values not sharing common superscript letter $(s)$ in a column are significantly different at $P<0.05$.

The results of the concentrations of phenols are presented in the Table 5. Total phenol ranked highest among the phytocheemicals determined. The mean phenol concentrations recorded were $2.64 \pm 0.15 \%, 2.40 \pm 0.34 \%, 2.62 \pm 0.31 \%, 2.50 \pm 0.16 \%$ and $2.80 \pm 0.08 \%$ for groundnut, millet, wheat, guinea corn and breadfruit, respectively. The highest concentration of $2.90 \%$ was obtained from breadfruit from Abia State while the lowest concentration $(2.00 \%)$ was recorded for millet from Imo State. The mean concentrations of phenol differed significantly $(\mathrm{P}<0.05)$ among the food items studied with breadfruit having the highest phenol content.

The levels of phytic acid in the grain samples investigated are presented in the Table 6 . Groundnut had the highest concentration (mean=0.30 \pm 0.03 ) of phytic acid compared to the other food items studied. The results obtained were lower than those reported by Simwemba et $\mathrm{al}^{18}$., However, Kheterpaul and Chauhan ${ }^{19}$ reported very high levels of $990 \mathrm{mg} \mathrm{g}_{-}{ }^{1}$ while $\mathrm{Oke}^{20}$ reported $532 \mathrm{mg} \mathrm{g}^{1}{ }^{1}$ for Nigerian pearl millet. Variations in phytic acid content among different food items can be attributed to both genetic and environmental conditions ${ }^{18}$. The results in Table 6 indicate moderate levels of phytic acid in the food grains studied. However, dietary phytic acid has been reported to inhibit intestinal absorption of certain divalent minerals such as $\mathrm{Ca}$ and $\mathrm{Mg}^{17}$, limit protein and starch digestibility ${ }^{21,22}$, hinder mineral bioavailability ${ }^{23}$ and inhibit proteolytic ${ }^{24}$ and amylolytic ${ }^{25}$ enzymes. 
Table 5. Phenol content of grains, (\%).

\begin{tabular}{cccccc}
\hline State & Groundnut & Millet & Wheat & Guinea corn & Bread fruit \\
\hline Abia & $2.70^{\mathrm{af}} \pm 0.20$ & $2.72^{\mathrm{af}} \pm 0.11$ & $2.74^{\mathrm{ae}} \pm 0.23$ & $2.50^{\mathrm{bg}} \pm 0.21$ & $2.90^{\mathrm{a}} \pm 0.03$ \\
Imo & $2.68^{\mathrm{af}} \pm 0.02$ & $2.00^{\mathrm{i}} \pm 0.04$ & $2.82^{\mathrm{ac}} \pm 0.70$ & $2.49^{\mathrm{bg}} \pm 0.36$ & $2.70^{\mathrm{af}} \pm 0.08$ \\
Akwa Ibom & $2.40^{\mathrm{eh}} \pm 0.20$ & $1.98^{\mathrm{i}} \pm 0.09$ & $2.60^{\mathrm{ag}} \pm 0.40$ & $2.40^{\mathrm{eh}} \pm 0.07$ & $2.81^{\mathrm{ac}} \pm 0.01$ \\
Cross River & $2.70^{\mathrm{af}} \pm 0.20$ & $2.09^{\mathrm{hi}} \pm 0.03$ & $2.60^{\mathrm{ag}} \pm 0.20$ & $2.40^{\mathrm{eh}} \pm 0.20$ & $2.81^{\mathrm{ac}} \pm 0.03$ \\
Rivers & $2.70^{\mathrm{af}} \pm 0.20$ & $2.43^{\mathrm{ag}} \pm 0.23$ & $2.60^{\mathrm{ag}} \pm 0.01$ & $2.46^{\mathrm{cg}} \pm 0.05$ & $2.83^{\mathrm{ac}} \pm 0.02$ \\
Bayelsa & $2.52^{\mathrm{bg}} \pm 0.08$ & $2.24^{\mathrm{gi}} \pm 0.07$ & $2.83^{\mathrm{ab}} \pm 0.25$ & $2.64^{\mathrm{af}} \pm 0.04$ & $2.82^{\mathrm{ac}} \pm 0.15$ \\
Delta & $2.68^{\mathrm{af}} \pm 0.02$ & $2.56^{\mathrm{ad}} \pm 0.11$ & $2.61^{\mathrm{dg}} \pm 0.11$ & $2.61^{\mathrm{bg}} \pm 0.01$ & $2.75^{\mathrm{ae}} \pm 0.04$ \\
Edo & $2.70^{\mathrm{af}} \pm 0.01$ & $2.77^{\mathrm{ad}} \pm 0.08$ & $2.43^{\mathrm{dg}} \pm 0.07$ & $2.50^{\mathrm{bg}} \pm 0.10$ & $2.75^{\mathrm{ae}} \pm 0.05$ \\
Ondo & $2.69^{\mathrm{af}} \pm 0.01$ & $2.82^{\mathrm{ac}} \pm 0.13$ & $2.37^{\mathrm{fh}} \pm 0.32$ & $2.50^{\mathrm{bg}} \pm 0.06$ & $2.80^{\mathrm{ac}} \pm 0.03$ \\
Mean & $2.64^{\mathrm{b}} \pm 0.15$ & $2.40^{\mathrm{d}} \pm 0.34$ & $2.62^{\mathrm{b}} \pm 0.31$ & $2.50^{\mathrm{c}} \pm 0.16$ & $2.80^{\mathrm{a}} \pm 0.08$ \\
\hline
\end{tabular}

Values are mean $\pm S$.D of three determinations. Values not sharing common superscript letter $(s)$ in a column are significantly different at $P<0.05$.

Table 6. Phytic acid content of grains, (\%).

\begin{tabular}{cccccc}
\hline State & Groundnut & Millet & Wheat & Guinea corn & Bread fruit \\
\hline Abia & $0.30^{\mathrm{ac}} \pm 0.02$ & $0.18^{\mathrm{eh}} \pm 0.0$ & $0.29^{\mathrm{bc}} \pm 0$. & $0.11^{\mathrm{ij}} \pm 0.01$ & $0.19^{\mathrm{eg}} \pm 0.0$ \\
Imo & $0.33^{\mathrm{ab}} \pm 0$. & $0.29^{\mathrm{bc}} \pm$ & $0.26^{\mathrm{cd}} \pm 0$. & $0.12^{\mathrm{hj}} \pm 0.03$ & $0.15^{\mathrm{fj}} \pm 0.0$ \\
Akwa Ibom & $0.28^{\mathrm{bc}} \pm 0$. & $0.26^{\mathrm{cd}} \pm 0.0$ & $0.28^{\mathrm{bc}} \pm 0$. & $0.18^{\mathrm{eh}} \pm 0.04$ & $0.10^{\mathrm{j}} \pm 0.02$ \\
Cross River & $0.26^{\mathrm{cd}} \pm 0$. & $0.18^{\mathrm{eh}} \pm 0.0$ & $0.33^{\mathrm{ab}} \pm 0$. & $0.18^{\mathrm{eh}} \pm 0.05$ & $0.19^{\mathrm{eg}} \pm 0.0$ \\
Rivers & $036^{\mathrm{a}} \pm 0.03$ & $0.20^{\mathrm{ef}} \pm 0.0$ & $0.28^{\mathrm{bc}} \pm 0$. & $0.19^{\mathrm{eg}} \pm 0.05$ & $0.14^{\mathrm{fi}} \pm 0.0$ \\
Bayelsa & $0.29^{\mathrm{bc}} \pm 0$. & $0.15^{\mathrm{ej}} \pm 0.0$ & $0.30^{\mathrm{ac}} \pm 0.0$ & $0.19^{\mathrm{gi}} \pm 0.02$ & $0.19^{\mathrm{ei}} \pm 0.0$ \\
Delta & $0.28^{\mathrm{bc}} \pm 0$. & $0.17^{\mathrm{eh}} \pm 0.0$ & $0.31^{\mathrm{ac}} \pm 0.0$ & $0.19^{\mathrm{gi}} \pm 0.02$ & $0.19^{\mathrm{ei}} \pm 0.0$ \\
Edo & $0.27^{\mathrm{bd}} \pm 0$. & $0.29^{\mathrm{bc}} \pm 0.0$ & $0.30^{\mathrm{ac}} \pm 0.0$ & $0.29^{\mathrm{bc}} \pm 0.02$ & $0.20^{\mathrm{ef}} \pm 0.0$ \\
Ondo & $0.29^{\mathrm{bc}} \pm 0$. & $0.19^{\mathrm{eg}} \pm 0.0$ & $0.27^{\mathrm{bc}} \pm 0$. & $0.21^{\mathrm{de}} \pm 0.03$ & $0.18^{\mathrm{eh}} \pm 0.0$ \\
Mean & $0.30^{\mathrm{a}} \pm 0.0$ & $0.21^{\mathrm{b}} \pm 0.0$ & $0.29^{\mathrm{a}} \pm 0.0$ & $0.18^{\mathrm{c}} \pm 0.06$ & $0.17^{\mathrm{c}} \pm 0.0$ \\
\hline
\end{tabular}

Values are mean $\pm S$.D of three determinations. Values not sharing common superscript letter $(s)$ in a column are significantly different at $P<0.05$. 


\section{Conclusion}

This study has revealed that some of the grains studied contained substantial amounts of some of the phytochemicals examined.

\section{References}

1. Bressani R, Food Rev Int., 1993, 9, 217-297.

2. Philips R D and McWaters K M, Food Technol., 1991, 45, 127-130.

3. Ahmed M B, hamed R A, Ali M E, Hassan A B and Babiker E E, Pakistan J Nutr., 2006, 5(5), 481-484.

4. Aletor V A and Adeogun O A, Food Chem., 1995, 53, 375-379.

5. Harborn J B, Phytochemical methods: A guide to modern techniques of plant analysis, Chapman and Hall New York, 1973, 185-190.

6. Hang W and Lantzsch H J, J Sci Food Agric., 1983, 34, 1423-1426.

7. Obadoni B O and Ochuko P O, Global J Pure Applied Sci., 2002, 8, 203-208.

8. Bohm B A and Kocipai-Abyazan M R, Pacific Sci., 1994, 48, 458-463.

9. Price M L and Butler L G, J Agric Food Chem., 1977, 25, 1268-1273.

10. Pearson D, The chemical analysis of foods, $7^{\text {th }}$ Ed., Churchill Livingstone, London, 1976.

11. Hertog M G L, Feskeens E J M, Hokman C H and Katan A, Lancet, 1993, 342, 1007-1011.

12. Kandaswami C, Perkins E, Soloniuk D S, Arzewiecki G and Middle E, Cancer Lett., 1991, 56, 147-152.

13. Hirono T, Gotch M and Oka K, Life Sci., 1994, 55, 1061-1069.

14. Elangovan V, Sekar N and Gorindasamy S, Cancer Lett., 1994, 87, 107-113.

15. Nakayama N G, Lindsey M L and Michael L H, Antiviral Res., 1993, 21, 289-299.

16. Jimoh F O and Oladiji A T, Afr J Biotechnol., 2005, 4(12), 1439-1442.

17. Osagie U and Eka U O, Post Harvest Res., 1998, 271.

18. Simwemba C G, Goseney R C, Varriano-Marston E and Zeleznak K, J Agric Food Chem., 1984, 32, 31-34.

19. Kheterpaul N and Chauhan B M, J Sci Food Agric., 1991, 55, 1080-1082.

20. Oke O L, Indian J Med Res., 1965, 53, 417-421.

21. Yoon J H, Thompson L U and Jenkins D J A, American J Clin Nutr., 1983, 38, 481-493.

22. Carnovali E, Lugaro E Lombardi-Boccia G, Cereal Chem., 1988, 65, 114-117.

23. Harland B F and Oberlease D, World Nutr Diet., 1987, 52, 235-259.

24. Knuckles B E, Kuzmicky D D and Betschart A A, J Food Sci., 1985, 52, 1080-1082.

25. Sharma C B, Goel M and Irshad M, Phytochemistry, 1978, 17, 201-204. 


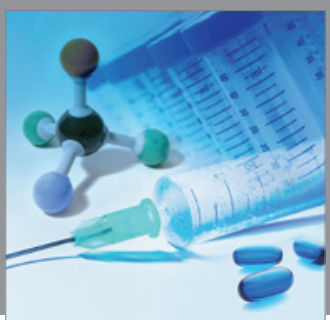

International Journal of

Medicinal Chemistry

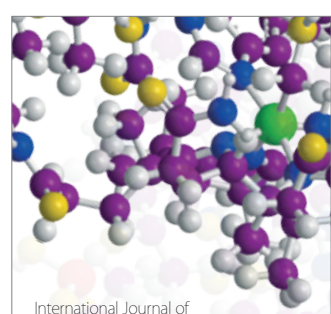

Carbohydrate Chemistry

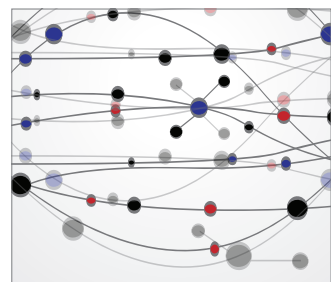

The Scientific World Journal
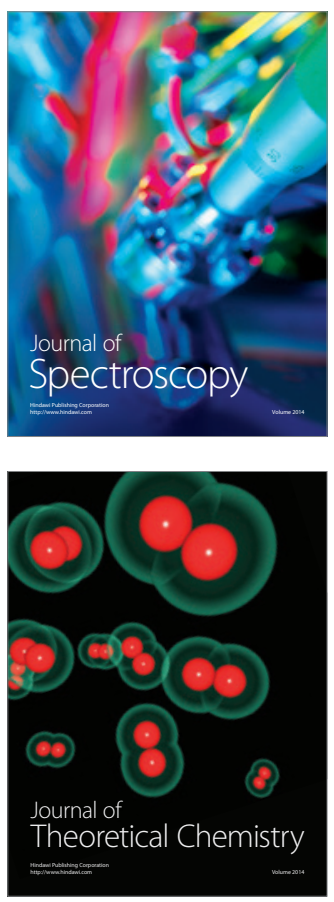
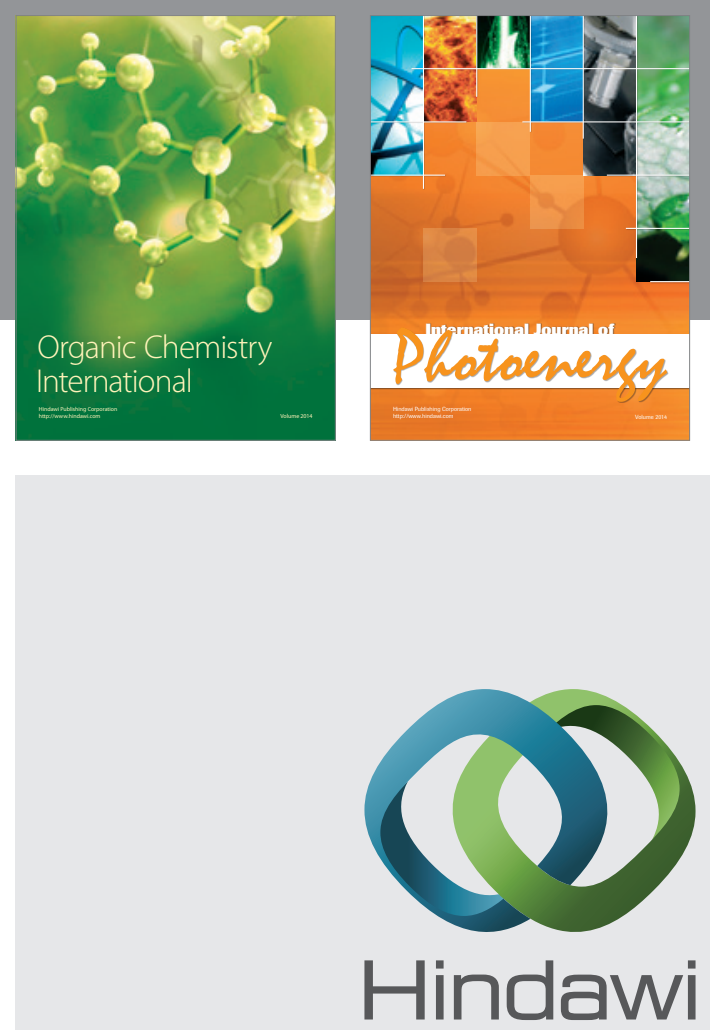

Submit your manuscripts at

http://www.hindawi.com
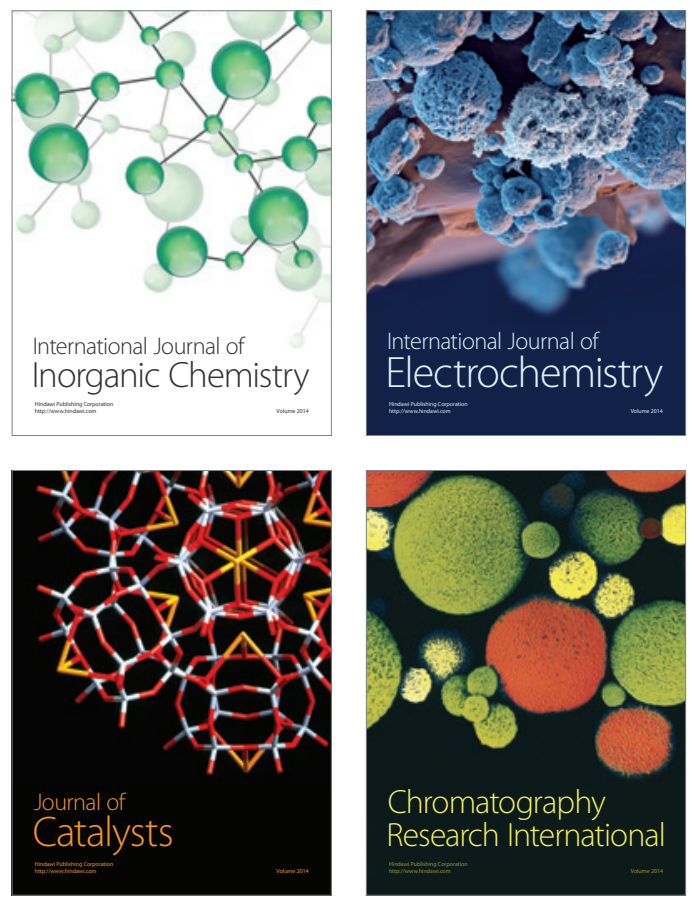
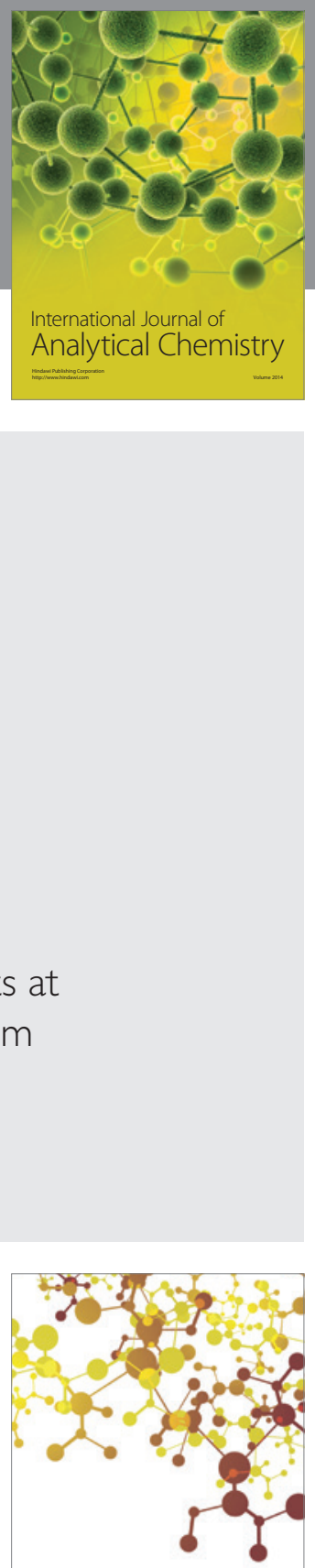

Journal of

Applied Chemistry
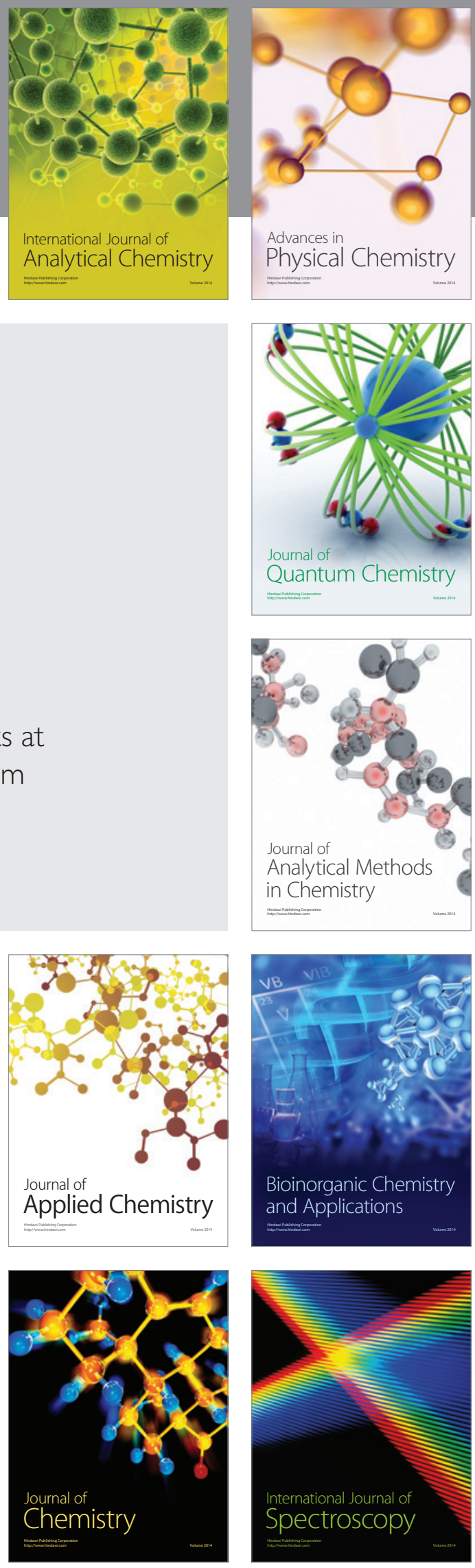